\title{
RENORMING DUAL BANACH LATTICES
}

\author{
N. GHOUSSOUB
}

\begin{abstract}
We show that a Banach lattice is order continuous as well as its dual if and only if it admits an equivalent Fréchet differentiable and locally uniformly convex lattice norm such that its dual norm is also locally uniformly convex.
\end{abstract}

Introduction. A norm \| \| on a Banach space is said to be locally uniformly convex if $\left\|x_{n}\right\| \rightarrow\|x\|$ and $\left\|x+x_{n}\right\| \rightarrow 2\|x\|$ imply that $x_{n} \rightarrow x$ strongly. Kadec showed that every separable Banach space has an equivalent uniformly convex norm [2]. Trojanski extended this to all weakly compactly generated spaces [2]. Recently, L. Vasak [8] extended it to all weakly $K$-analytic Banach spaces. A characterization of Banach spaces which are locally uniformly convexifiable is still an open problem.

A norm \| \| on a Banach space $E$ is said to be Fréchet differentiable if, for every $x$ in $E, \lim _{\lambda \rightarrow 0}(\|x+\lambda y\|-\|x\|) / \lambda$ exists uniformly for $y$ in the unit sphere of $E$. Kadec and Klee [2] showed that a separable Banach space with a separable dual admits an equivalent norm which is locally uniformly convex, Fréchet differentiable and such that its dual norm is also uniformly convex. John and Zizler [2] extended this result to weakly compactly Banach spaces (W.C.G.) having also W.C.G. duals.

In the case of a Banach lattice, it is natural to require that the new equivalent norm be also a lattice norm $(|x| \leqslant|y| \Rightarrow\|x\| \leqslant\|y\|)$. In this direction Davis, Ghoussoub and Lindenstrauss showed that a Banach lattice $E$ has an equivalent locally uniformly convex lattice norm if and only if $E$ has an order continuous norm [1]. Aside from yielding lattice renormings, the methods of [1] imply the existence of equivalent locally uniformly convex norms on some spaces which are not "small" in the sense of separable or weakly compactly generated, provided, of course, these spaces are equipped with a lattice structure.

In this note, we give a lattice analog to the result of John and Zizler, that is a Banach lattice has an order continuous norm as well as its dual if and only if it has an equivalent locally uniformly convex lattice norm, Fréchet differentiable, such that its dual norm is also locally uniformly convex. For more details about Banach lattices, we refer to the book of Lindenstrauss and Tzafriri [5].

We start by giving the following easy lemma which shows that Asplund's averaging technique conserves the lattice property of the averaged norms.

Received by the editors April 23, 1980.

AMS (MOS) subject classifications (1970). Primary 46B99, 60G99.

Key words and phrases. Banach lattice local uniform convexity, renorming.

() 1982 American Mathematical Society 0002-9939/81/0000-1089/\$02.00 
LEMMA. If $f_{0}$ and $g_{0}$ are two lattice norms on Banach lattice $E$, then the norms defined by

$$
f_{1}=\frac{f_{0}+g_{0}}{2} \text { and } g_{1}(x)=\inf \left\{\frac{f_{0}(x+y)+g_{0}(x-y)}{2}, y \in E\right\}
$$

are also lattice.

Proof. The norm $f_{1}$ is trivially lattice. For $g_{1}$, notice that

$$
g_{1}(x)=\inf \left\{f_{0}(t)+g_{0}(x-t) ; t \in E\right\} .
$$

We want to show that the infimum may be taken over the $t$ 's in the order interval $[0, x]$. For that, suppose $x$ in $E_{+}$; then, since $f_{0}$ and $g_{0}$ are lattice norms, we have

$$
\begin{aligned}
g_{1}(x) & =\inf \left\{f_{0}(|t|)+g_{0}(|x-t|) ; t \in E\right\} \\
& \geqslant \inf \left\{f_{0}(|t|)+g_{0}(|x-| t||) ; t \in E\right\} \\
& =\inf \left\{f_{0}(t)+g_{0}(|x-t|) ; t \in E_{+}\right\} \\
& \geqslant \inf \left\{f_{0}(t \wedge x)+g_{0}(x-t \wedge x) ; t \in E_{+}\right\} \\
& =\inf \left\{f_{0}(s)+g_{0}(x-s) ; 0<s<x\right\} .
\end{aligned}
$$

Suppose now $0 \leqslant x \leqslant x^{\prime}$. For every $t, 0 \leqslant t \leqslant x^{\prime}$, we have

$$
f_{0}(t \wedge x)+g_{0}(x-t \wedge x) \leqslant f_{0}(t)+g_{0}\left(x^{\prime}-t\right) \text {. }
$$

It follows that $g_{1}(x) \leqslant g_{1}\left(x^{\prime}\right)$.

The main result is the following.

THEOREM. If $E$ is a Banach lattice, the following properties are equivalent:

(1) $E$ and $E^{*}$ have order continuous norms.

(2) There exists an equivalent lattice norm on $E$ which is locally uniformly convex, Fréchet differentiable and such that its dual norm is also locally uniformly convex on $E^{*}$.

Proof. $(1) \Rightarrow(2)$. Since $E$ has an order continuous norm, there exists by Theorem I.2 of [1] an equivalent lattice norm $p_{1}$ which is locally uniformly convex. For the dual $E^{*}$, we are going to use the same construction of the norm as in [1], but be more careful to insure that the new norm is weak star lower semicontinuous.

Suppose first that $E^{*}$ has a weak unit $e^{*}$. We claim that $E$ also has a weak unit $e$. If not, then there exists in $E$ a normalized uncountable family $\left(x_{\alpha}\right)_{\alpha \in \Gamma}$ of positive pairwise disjoint elements. Since $e^{*}\left(x_{\alpha}\right)$ is strictly positive for every $\alpha$ in $\Gamma$, there exist $\delta>0$ and an uncountable subset $\Gamma^{\prime}$ of $\Gamma$ such that $e^{*}\left(x_{\alpha}\right)>\delta$ for all $\alpha$ in $\Gamma^{\prime}$. It is easily seen that the closed linear space of $\left(x_{\alpha}\right)_{\alpha \in \Gamma^{\prime}}$ is a sublattice of $E$, lattice isomorphic to $l^{1}\left(\Gamma^{\prime}\right)$, which is a contradiction.

Consider now $\left(E^{*}, e\right)$, the completion of $E^{*}$ equipped with the $L$-norm $\left\|x^{*}\right\|_{1}=$ $e\left(\left|x^{*}\right|\right)$. It is an $A-L$ space; hence there exists a probability space $(\Omega, \mathscr{F}, P)$ such that $\left(E^{*}, e\right)$ is lattice isomorphic to $L^{1}(\Omega, \mathscr{F}, P)$. Since the order interval $[-e, e]$ is weakly compact, the dual of $\left(E^{*}, e\right)$ is the $A-M$ space $E_{e}$ which is the ideal generated by $e$. It follows that the inclusion map $E^{*} \rightarrow L^{1}(\Omega, \mathscr{F}, P)$ is weak star to weak continuous [7, p. 114]. 
For every $n$ and every $f$ in $E^{*}$ define $\psi_{n}(f)=\sup _{P(A)<1 / n}\left\|f \chi_{1}\right\|_{E^{*}}$. The norm $\psi_{n}$ is weak star lower semicontinuous, since $f \rightarrow f \chi_{1}$ is a projection band in $E^{*}$, hence weak star continuous. Define now on $E^{*}$ the norm

$$
\||f|\|=\left\|\left(\|f\|_{E^{*}},\|f \mid\|_{1}, \psi_{1}(f), \psi_{2}(f), \ldots\right)\right\|_{\tilde{c}_{0}}
$$

where $\|f\|_{1}$ is the locally uniformly convex lattice norm constructed in [1] on $L^{1}(\Omega, \mathscr{F}, P)$ and $\tilde{c}_{0}$ is the Banach lattice $c_{0}$ equipped with Day's norm [2, p. 94]. By the above ||| ||| is weak star lower semicontinuous, and by [1] it is locally uniformly convex and lattice.

Suppose now $E^{*}$ does not have a weak unit. Let $\left(e_{\alpha}^{*}\right)_{\alpha \in \Gamma}$ be a maximal orthogonal system in $E^{*}$, that is $E^{*}=\Sigma_{\alpha \in \Gamma} \oplus E_{\alpha}^{*}$ where each $E_{\alpha}^{*}$ is the closed ideal generated by $e_{\alpha}^{*}$. Since $E$ has an order continuous norm, $E_{\alpha}^{*}$ is weak star closed; hence $E_{\alpha}^{*}$ is the dual of $E / E_{\alpha}^{* 0}$ where $E_{\alpha}^{* 0}$ is the polar of $E_{\alpha}^{*}$ (bipolar theorem). By the above, $E / E_{\alpha}^{* 0}$ has a weak unit which if "lifted" to $E$ gives an element $e_{\alpha}$ in $E$, strictly positive on $E_{\alpha}^{*}$. Let now $L^{1}\left(\Omega_{\alpha}, \mathscr{F}_{\alpha}, P_{\alpha}\right)$ be the space associated to the functional $e_{\alpha}$ : We get that the inclusion map $E_{\alpha}^{*} \rightarrow L^{1}\left(\Omega_{\alpha}, \mathscr{F}_{\alpha}, P_{\alpha}\right)$ is weak star to weak continuous and from the first part of the proof, we may construct a locally uniformly convex lattice norm $\|\mid\|_{\alpha}$ on $E_{\alpha}^{*}$ which is weak star lower semicontinuous.

We proceed again as in [1]; let $Q_{\alpha}$ denote the band projection of $E^{*}$ onto $E_{\alpha}^{*}$. It is weak star continuous. Therefore

$$
G_{n}(f)=\sup \left\{(\operatorname{card} A) \sum_{\alpha \in A}\left\|Q_{\alpha} f\right\|_{\alpha}+\left\|\left(I-\sum_{\alpha \in A} Q_{\alpha}\right) f\right\|_{E^{*}} ; A \subseteq \Gamma, \operatorname{card} A=n\right\}
$$

is weak star lower semicontinuous and the norm defined by

$$
\|f\|\|=\|\left(\|f\|_{E^{*}},\left(2^{-n} G_{n}(f)\right)_{n},\left(\left\|\mid Q_{\alpha} f\right\|_{\alpha}\right)_{\alpha \in \Gamma}\right) \|_{\tilde{c}_{0}(\{0\} \cup N \cup \Gamma)}
$$

is weak star lower semicontinuous. It follows that there exists a lattice norm $p_{2}$ on $E$ such that its dual norm is $\left\||\||\right.$ which is locally uniformly convex; hence $p_{2}$ is Fréchet differentiable. Now, apply Asplund's averaging technique to $p_{1}$ and $p_{2}$ and the lemma to get the required norm [2, p. 106].

The converse follows immediately from [1].

Corollary. A Banach lattice is reflexive if and only if it can be given an equivalent lattice norm such that both the space and its dual are simultaneously locally uniformly convex and Fréchet differentiable.

Half of this follows from the theorem above; the converse depends on a theorem of V. L. Smul'yan [2, p. 34] that if $E^{*}$ has a Fréchet differentiable norm, then $E$ is reflexive.

Remarks. A well-known result of Ekeland and Lebourg [4] asserts that if a Banach space has a Fréchet differentiable equivalent norm, then it is an Asplund space. The converse for this result is still an open problem. It is also well known that if a dual Banach space can be renormed dually in a locally uniformly convex manner then it has the Radon-Nikodym property. Recently, G. A. Edgar [3] constructed the "long James space", which is a dual Banach space with the 
Radon-Nikodym property, but cannot be renormed dually to be locally uniformly convex. But it is easy to see that this space is not a Banach lattice. Therefore the following question remains open: Is every dual Banach lattice with the RadonNikodym property renormable to be a dual locally uniformly convex Banach lattice? The above theorem gives a positive answer to this question when the predual $E$ is countably order complete and $E^{*}$ has the Radon-Nikodym property, but the weak star lower semicontinuity of the constructed norm relies heavily on $E$ being order continuous. One way of trying to use the construction above is to show that if a dual Banach lattice has the Radon-Nikodym property, then it has at least one order continuous predual. This question coincides with a problem asked by Lotz [6] and which was answered negatively by a counterexample of Talagrand [9].

\section{REFERENCES}

1. W. Davis, N. Ghoussoub and J. Lindenstrauss, $A$ lattice renorming theorem and applications to vector valued processes, Trans. Amer. Math. Soc. 263 (1981), 531-540.

2. J. Diestel, Geometry of Banach spaces. Selected topics, Springer-Verlag, Berlin, Heidelberg and New York, 1975.

3. G. A. Edgar, The long James space (to appear).

4. I. Ekeland and G. Lebourg, Generic Fréchet differentiability and perturbed optimization problems in Banach spaces, Trans. Amer. Math. Soc. 224 (1977), 193-216.

5. J. Lindenstrauss and L. Tzafriri, Classical Banach spaces. Function spaces, vol. II, Springer-Verlag, Berlin and New York, 1979.

6. H. P. Lotz, The Radon-Nikodym property in Banach lattices, Univ. of Illinois, Urbana-Champaign, preprint, 1975.

7. H. H. Shaefer, Banach lattices and positive operators, Springer-Verlag, Berlin, Heidelberg and New York, 1975.

8. L. Vasak, On a generalisation of W.C.G. Banach spaces, Studia Math. (to appear).

9. M. Talagrand, Dual Banach lattices and Banach lattices with the Radon-Nikodym property, preprint, 1980.

Department of Mathematics, University of British Columbia, Vancouver, Brttish Columbia, V6T 1Y4 CaNada 